\title{
A General Method for Quantitative Measurement of Molecular Mass Distribution by Mass Spectrometry
}

\author{
E. S. Park, W. E. Wallace, C. M. Guttman, K. M. Flynn, \\ M. C. Richardson, and G. A. Holmes \\ Polymers Division, National Institute of Standards and Technology, Gaithersburg, Maryland, USA
}

A method is presented to test whether the conversion of the mass spectrum of a polydisperse analyte to its molecular mass distribution is quantitative. Mixtures of samples with different average molecular masses, coupled with a Taylor's expansion mathematical formalism, were used to ascertain the reliability of molecular mass distributions derived from mass spectra. Additionally, the method describes how the molecular mass distributions may be corrected if the degree of mass bias is within certain defined limits. This method was demonstrated on polydisperse samples of $\mathrm{C}_{60}$ fullerenes functionalized with ethylpyrrolidine groups measured by matrix-assisted laser desorption/ionization time-of-flight mass spectrometry; however, it is applicable to any polydisperse analyte and mass spectrometric method as long as spectrum resolution allows individual oligomers to be identified. Mass spectra of the derivatized fullerenes taken in positive ion mode were shown to give an accurate measurement of the molecular mass distribution while those taken in negative ion mode were not. Differences in the mechanisms for ion formation are used to explain the discrepancy.

Official contribution of the National Institute of Standards and Technology; not subject to copyright in the United States of America. (J Am Soc Mass Spectrom 2009, 20, 1638-1644) Published by Elsevier Inc. on behalf of American Society for Mass Spectrometry

$\mathrm{M}$ ass spectrometry is often used to determine the molecular mass distribution (MMD) of polydisperse analytes, such as industrial polymers, functionalized nanoparticles, or synthetic polyamino acids. However, the analyst must exercise caution in interpreting the mass spectra of polydisperse materials. It is well documented that differences in ion creation, separation, and detection as a function of oligomer mass can lead to mass spectra which, without a correct interpretation, give misleading molecular mass distributions. As noted in the classic text by McLafferty and Tureček [1] in their discussion of mixture analysis, "Spectral superposition will not necessarily be linear for chemical ionization and other spectra obtained at pressures high enough for competitive ion-molecule reactions." Competitive ion-molecule reactions are found in many modern ionization methods, most notably in matrix-assisted laser desorption/ionization (MALDI) and electrospray ionization (ESI).

To overcome the challenge of finding the proper molecular mass distribution for polydisperse materials from their mass spectra in cases where individual oligomers of the polydisperse material are not available,

Address reprint requests to Dr. W. E. Wallace, Polymers Division, National Institute of Standards and Technology, 100 Bureau Drive stop 8541, Gaithersburg, MD 20899-8541 USA. E-mail: william.wallace@nist.gov a quantitative signal intensity calibration method has been created by Guttman et al. [2,3], and used to create an absolute molecular mass distribution polymer standard. Their method uses gravimetric mixtures of polydisperse analytes with different average molecular masses, coupled with a Taylor's expansion mathematical formalism, to develop a calibration curve to correct the ion intensities in the mass spectrum. This leads to a more reliable measure of the molecular mass distribution. A streamlined approach to their Taylor's expansion method is presented here.

To demonstrate how this new method works the functional group distribution of multi-substituted fulleropyrrolidines was measured using MALDI time-offlight (TOF) mass spectrometry. The grafting of organic functionalities onto organic or inorganic nanoparticles is used routinely to manipulate their physical, chemical, or biologic behavior. In particular, fullerene chemistry is one of the most developed areas of nanoparticle functionalization [4,5]. Measurements of the degree of derivatization of fullerenes by mass spectrometry have been undertaken previously by many groups, starting with the work of Bausch et al. in 1991 on methylated fullerenes [6]. However, previous studies lacked a methodology to insure that the mass spectra presented are truly indicative of the molecular mass distribution of the functionalized fullerene. 


\section{Experimental}

\section{Materials}

$\mathrm{N}$-ethylpyrrolidine $\mathrm{C}_{60}$ fullerene derivatives were synthesized using the well-known Prato reaction, shown in Scheme 1, which generates reactive azomethine ylides through the condensation of a $\alpha$-amino acid and an aldehyde, and results in 1,3-dipolar cycloaddition of compounds [7-9]. MALDI-TOF mass spectra in negative and in positive ion modes were taken using trans, trans-1,4-diphenyl-1,3-butadiene(DPB;CASnumber 88665-7), as a matrix. Chloroform was used as the solvent for the MALDI target preparation.

\section{N-Ethylpyrrolidine $C_{60}$ Fullerene Derivative A: Low Degree of Functionalization}

A mixture of $108 \mathrm{mg}$ of $\mathrm{N}$-ethylglycine and $1000 \mathrm{mg}$ of paraformaldehyde was heated for $2 \mathrm{~h}$ at reflux in $80 \mathrm{~mL}$ of toluene in the presence of $200 \mathrm{mg}$ of $\mathrm{C}_{60}$. The resulting brown solution was reduced by rotary evaporator and purified using silica gel flash chromatography (730 mesh silica, eluent: toluene, then chloroform and triethylamine in a 10:1 by volume fraction mixture).

\section{$N$-Ethylpyrrolidine $C_{60}$ Fullerene Derivative B: High Degree of Functionalization}

A mixture of $2000 \mathrm{mg}$ of $\mathrm{N}$-ethylglycine and $4000 \mathrm{mg}$ of paraformaldehyde was heated at reflux in $200 \mathrm{~mL}$ of toluene in the presence of $500 \mathrm{mg}$ of $\mathrm{C}_{60}$. The reaction proceeded overnight. The resulting brown solution was reduced by rotary evaporator and purified using silica gel flash chromatography (730 mesh silica, eluent: toluene, then chloroform and triethylamine in a 10:1 by volume fraction mixture).

\section{Instrumentation}

Mass spectrometry was performed on a Bruker Daltonics (Billerica, MA, USA) REFLEX time-of-flight instrument with delayed extraction. Initially, reflectron mode ion separation was used to identify species because of its higher mass resolution; however, linear mode is preferred for quantitation because of the chance that oligomers with different numbers of functional groups may undergo metastable fragmentation at different rates. This would lead to inaccurate peak areas and cannot be accounted for without measuring the metastable decay rates of the different oligomers. All spectra used for quantitative analysis, as well as those spectra shown in the figures, were taken in linear mode. Ions were generated with a 337-nm wavelength nitrogen gas laser an average energy of $\sim 0.3 \mu \mathrm{J}$ per pulse spread over a spot size of $100 \mu \mathrm{m}$ in diameter. A delay of 250 ns was used before ions were extracted from the laser plume. Mass calibration was performed daily with neat CsI, which forms abundant ion clusters of the form $\left[\mathrm{Cs}_{\mathrm{n}} \mathrm{I}_{\mathrm{m}}\right]^{+}$and $\mathrm{I}_{\mathrm{n}}^{-}$. Data analysis was performed using the Polymerix computer code (Sierra Analytics, Modesto, CA). Previous work has shown that the estimated standard uncertainty of the peak position from calibration and repeatability is $0.2 \mathrm{u}$ at $3000 \mathrm{u}$ and the estimated standard uncertainty in the overall signal intensity from repeatability studies is $15 \%$ [10]. For all experiments, a solution of $40 \mathrm{mg} / \mathrm{mL}$ DPB was mixed with a $5 \mathrm{mg} / \mathrm{mL}$ solution of the analyte in a volume ratio of 3:1. The solutions were electrosprayed [11] onto the stainless steel MALDI target and allowed to air dry.

\section{Theory and Numerical Methods}

\section{Mathematical Preliminaries}

This section describes the conceptual framework for the method using a simple model system. If the mass spectrometry experiments are conducted in a linear range of target concentration versus signal intensity for each oligomer (designated as $i=1,2,3$, etc.) then:

$$
S_{i}=k_{i} n_{i}
$$

where $k_{i}$ converts number of oligomers in the sample $n_{i}$ into signal intensity $S_{i}$ in the mass spectrum. Note that each oligomer must appear as an individual peak in the mass spectrum whose intensity can be measured, typically by peak integration. Similarly the total amount of each oligomer, $G_{i}$, can be expressed as:

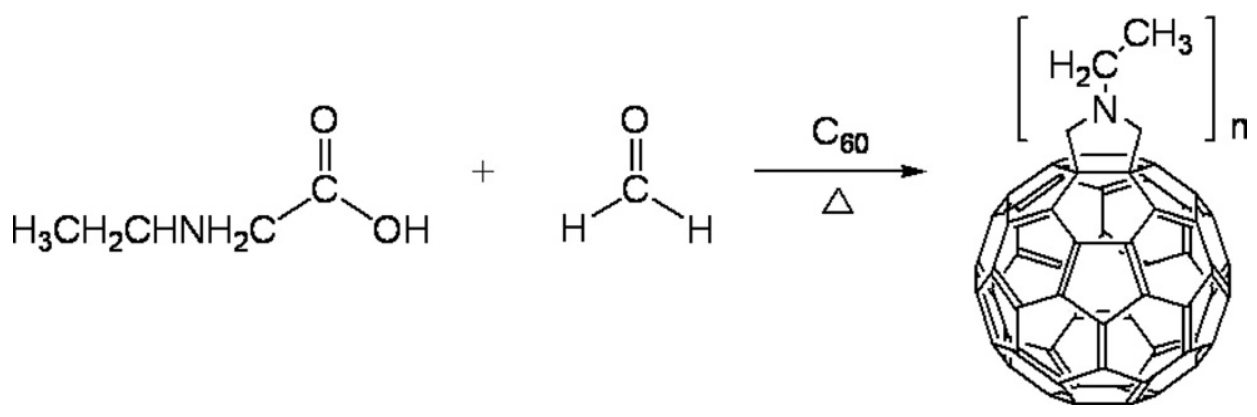

Scheme 1 


$$
G_{i}=m_{i} n_{i}
$$

Assume without loss of generality that each of the two components to be mixed (components are designated as $j=1,2,3$, etc.) consist of one species, that is, a single molecular mass. Another way to state this is that each component has a polydispersity (PD) equal to 1 . For such a gravimetric mixture (with mixtures designated as $q=\mathrm{A}, \mathrm{B}, \mathrm{C}$, etc.):

$$
S_{A}=k_{1} n_{1}+k_{2} n_{2}
$$

For gravimetric mixture A the mass-average molecular mass can be expressed as:

$$
M_{w A}=\frac{\left(k_{1} m_{1}^{2} n_{1}+k_{2} m_{2}^{2} n_{2}\right)}{\left(k_{1} m_{1} n_{1}+k_{2} m_{2} n_{2}\right)}
$$

or as:

$$
M_{w A}=\frac{\left(k_{1} m_{1} G_{1}+k_{2} m_{2} G_{2}\right)}{\left(k_{1} G_{1}+k_{2} G_{2}\right)}
$$

To simplify eq 5 let

$$
X=\frac{G_{1}}{G_{1}+G_{2}}
$$

Substituting eq 6 into eq 5 and dividing numerator and denominator by $\left(G_{1}+G_{2}\right)$ yields:

$$
M_{w A}=\frac{\left(k_{1} m_{1} X+k_{2} m_{2}(1-X)\right)}{\left(k_{1} X+k_{2}(1-X)\right)}=\frac{\left(m_{1} X+\theta m_{2}(1-X)\right)}{(X+\theta(1-X))}
$$

where

$$
\theta=k_{2} / k_{1}
$$

In this way the mass bias in the mass spectrum is reduced to a single metric, $\theta$. $\theta$ equals one for an unbiased system. If component 2 is overcounted with respect to component $1, \theta$ will be greater than one, if component 2 is undercounted, $\theta$ will be less than one. The further $\theta$ is from one the greater the bias in the mass spectrum.

\section{Extension to Polydisperse Analytes}

Equation 7 can be extended to a gravimetric mixture of polydisperse components by substituting the experimental average molecular mass of each pure component derived from its mass spectrum:

$$
\begin{aligned}
M_{w q}^{\text {grav }} & =\frac{\left(\hat{k}_{1} M_{w 1}^{\exp X}+\hat{k}_{2} M_{w 2}^{\exp }(1-X)\right)}{\left(\hat{k}_{1} X+\hat{k}_{2}(1-X)\right)} \\
& =\frac{\left(M_{w 1}^{\exp } X+\theta M_{w 2}^{\exp }(1-X)\right)}{(X+\theta(1-X))}
\end{aligned}
$$

where $\hat{k}_{1}$ and $\hat{k}_{2}$ replace $k_{1}$ and $k_{2}$ used in the monodisperse example and are the mass-average means over each component of the mixture, which is conceptually similar to the mass-average molecular mass. Likewise, $X$ is now calculated from the gravimetric amounts of each component in the mixture. The mass moments of the pure components are from their mass spectra using eq 10:

$$
M_{w q}^{\exp }=\sum_{i} S_{i q} m_{i}^{2} / \sum_{i} S_{i q} m_{i}
$$

To obtain an estimate of the value of $\theta$, the minimum value of the sum of squares is found. The sum of squares over all mixtures $q$ is expressed as:

$$
S S_{\theta}=\sum_{q}\left(M_{w q}^{\text {grav }}-M_{w q}^{\text {exp }}\right)^{2}
$$

The simplest way to solve this equation is to insert an arbitrary value for $\theta$ (typically $\theta=1$ ) and calculate a value for $S S_{\theta}$ then increment $\theta$ and recalculate $S S_{\theta}$. This most basic iterative process will yield an optimal value typically in a few steps and can easily be encoded in spreadsheet software. Recall that values of $\theta$ near one indicate systems with little bias in the mass spectrum.

\section{Calculating the Correction Factor for Each Oligomer}

Once $\theta$ has been calculated and found to be near one, the next step in the process is to calculate the various $k_{i}$ to correct the molecular mass distribution. If the $k_{i}$ are a smoothly and slowly varying function of $i$ (or $m_{i}$ ), a Taylor's expansion on $k_{i}$ may be made around a mass peak near the center of the MMD, termed $M_{0}$. The center is used to assure that the function is changing as little as possible over the entire width of the MMD; however, mathematically the choice is arbitrary. Thus:

$$
\begin{aligned}
& k_{i}=k_{0}+Q\left(m_{i}-M_{0}\right)+\text { higher order terms in } m_{i} \\
& S_{i}=k_{0} n_{i}+Q\left(m_{i}-M_{0}\right) n_{i}+\text { higher order terms in } m_{i}
\end{aligned}
$$

where $k_{0}$ and $Q$ are the first two coefficients in the Taylor's expansion. They are also functions of all the experimental conditions: the instrument parameters, the sample concentrations, and the sample preparation method. In this way the entire physics of the experiment is folded into these two coefficients. From these assumptions, and dropping the higher order terms in eq 13, one can derive [2] the following important relationship:

$$
M_{w q}^{\exp }=M_{w}^{0}\left\{\frac{1+\left(Q / k_{0}\right)\left(P D_{w} M_{w}^{0}-M_{0}\right)}{1+\left(Q / k_{0}\right)\left(M_{w}^{0}-M_{0}\right)}\right\}
$$

where $M_{w q}^{\exp }$ is the MALDI-TOF MS measured massaverage molecular mass for the mixture of analytes given in eq 10. $P D_{w}$ is mass average polydispersity 
$\left(M_{z}^{e x p} / M_{w}^{e x p}\right)$ and is taken here to be the experimentally measured value. Equation 14 is then solved for $M_{w}^{0}$ for various values of $Q / k_{o}$ at a fixed $M_{o}$ chosen as described below for the values of the mixtures described by $\mathrm{q}=$ A, B, C, etc. and for the initial components of the mixtures described as $\mathrm{j}=1$ and $\mathrm{j}=2$.

For a gravimetric mixture $A, M_{w A}^{\text {grav } 0}$ is calculated from the values for the individual components $M_{w 1}^{0}$ and $M_{w 2}^{0}$ computed for each $Q / k_{o}$ using a simple weighted average:

$$
M_{w A}^{\text {grav }}-0=\frac{G_{1}}{G_{1}+G_{2}} M_{w 1}^{0}+\frac{G_{2}}{G_{1}+G_{2}} M_{w 2}^{0}
$$

where $G_{1}$ is the gravimetric mass of component 1 in the mix, and $G_{2}$ is similarly defined.

For each $Q / k_{o}$ we compute the sum of squares, SS, as

$$
S S_{\left(\mathrm{Q} / k_{0}\right)}=\sum_{q}\left(M_{w q}^{g r a v \_0}-M_{w q}^{0}\right)^{2}
$$

where the sum is taken over all measured mixtures. The $Q / k_{0}$, which gives the minimum value of the SS, is then taken as the best fit. As with eq 11, solution of eq 16 requires iteration over incremented values of $Q / k_{0}$.

Dropping the higher order terms and rearranging eq (13) yields:

$$
\frac{S_{i}}{k_{0} n_{i}}=1+\frac{Q}{k_{0}}\left(m_{i}-M_{0}\right)
$$

Equation 17 shows us how to apply the correction factor $Q / k_{0}$ to each oligomer $m_{i}$ to arrive at a more reliable measure of the molecular mass distribution. If $Q / k_{0}$ were equal to zero, the mass spectrum would show no mass bias and $S_{i}=k_{0} n_{i}$, meaning that the peak areas are directly proportional to the oligomer concentrations in the sample. If $Q / k_{0}$ is non-zero, mass bias is present. If $\mathrm{M}_{0}$ is taken at the middle of the distribution, the sign of $Q / k_{0}$ along with the mass of an oligomer $m_{i}$ determines if the correction is positive or negative.

The steps of the method can be summarized as follows:

1. Obtain two samples having different molecular mass distributions but with otherwise identical properties. For example, these could be polymers with different degrees of polymerization or nanoparticles with different levels of functionalization.

2. Take mass spectra of each sample using the same instrument settings endeavoring to keep all experimental conditions constant.

3. Use a laboratory balance to make carefully controlled gravimetric mixtures of the two samples in several well-spaced ratios.

4. Take mass spectra of each mixture using the same instrument settings as used for the pure components. The instrument settings may not be optimal for the mixtures but they must be held constant to satisfy the self consistency of the method.
5. From the mass spectra calculate the mass-average molecular masses for the pure components and for the mixtures.

6. Use eq 11 to iteratively calculate the minimum value of $\theta$. If $\theta$ is between 0.5 and 2 , then the possibility exists that the molecular mass distribution can be corrected. If not, the results should be treated with caution, and the error is too great to be corrected using only the linear term in the Taylor's expansion.

7. Choose $\mathrm{M}_{0}$, a mass near the center of the average molecular masses of the two components. The exact choice of $\mathrm{M}_{0}$ is not critical; however, the correction to the distribution will be more accurate near $\mathrm{M}_{0}$ and less accurate the farther any given oligomer mass is from $\mathrm{M}_{0}$.

8. Use eq 16 to iteratively calculate $Q / k_{0}$.

9. Use eq 17 and the value for $Q / k_{o}$ to correct the ion intensities $S_{i}$ in the mass spectrum to arrive at a new molecular mass distribution.

\section{Results}

The mass spectra of the ethylpyrrolidine derivatives showed multi-substituted $\mathrm{C}_{60}$ with up to seven functional groups out of a hypothetical number of 9 . Figure 1 shows positive-ion MALDI-TOF mass spectra for the low functionalized material, the high functionalized material, and for three gravimetric mixtures of the two: 3:1, $2: 2,1: 3$. The ions are of the form $[\mathrm{M}+\mathrm{H}]^{+}$. Figure 2 shows negative-ion mass spectra taken on the same target immediately after the positive-ion mass spectra were taken. Here the ions are of the form $\left[\mathrm{M}^{*}\right]^{-}$. Each series of spectra were taken at the same laser energy per pulse. Inspection of Figures 1 and 2 shows early indications that mixing in the positive ion case gives good spectral

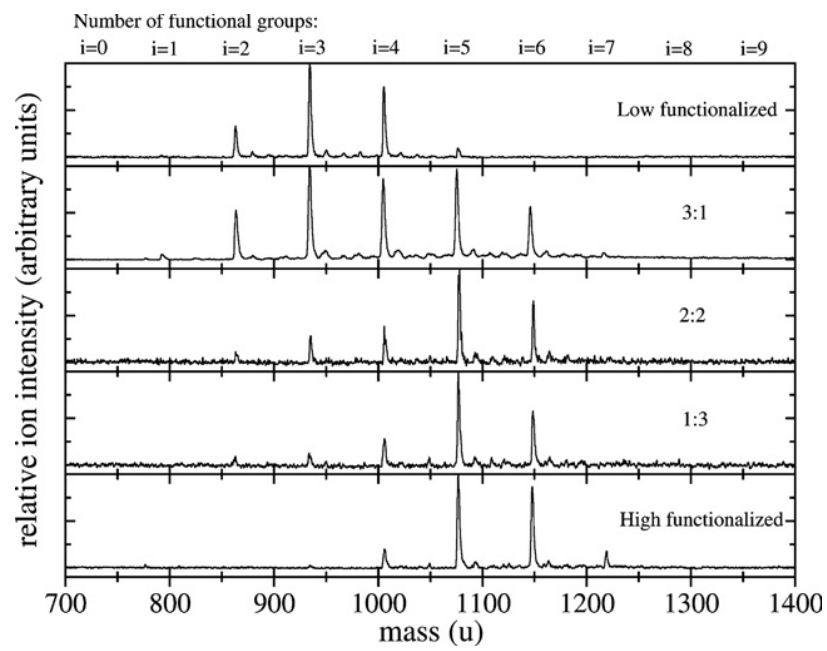

Figure 1. Positive ion mass spectra showing the low functionalized and the high functionalized components in the top and bottom panels, respectively, as well as three gravimetric mixtures of the two. Inspection of the middle panels points towards a measurement bias in favor of the more highly functionalized fullerenes. 


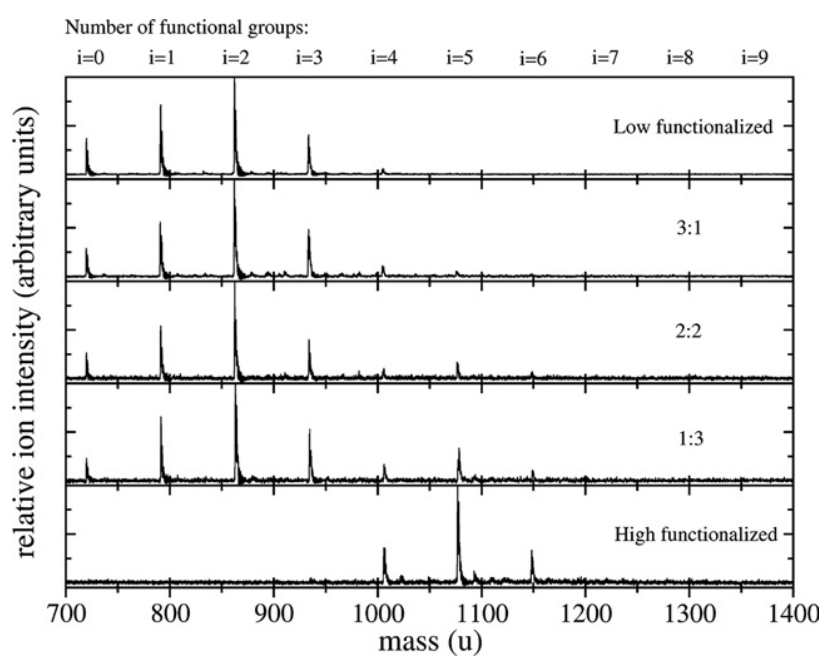

Figure 2. Negative ion mass spectra showing the low functionalized and the high functionalized components in the top and bottom panels, respectively, as well as three gravimetric mixtures of the two. Inspection of the middle panels points towards a strong measurement bias in favor of the less highly functionalized fullerenes.

superposition; however, in the negative ion case there is a strong bias toward the low mass ions.

Figure 3 shows the molecular mass moments calculated for the pure materials and each of the three mixtures for both positive and negative ions. Figure 3 validates the observations made on Figures 1 and 2. If there were no bias in the mass spectra, the molecular mass moments of the mixtures would simply be the weighted average of those of the pure compounds. If this were the case, all points would lie on the diagonal line $\mathrm{x}=\mathrm{y}$, that is, the mass spectral moment for a distribution would be equal to the gravimetric moment. (The moments for the pure components will of course lie on the line $\mathrm{x}=\mathrm{y}$ ). Figure 3 shows that this is not the case. A measurement bias is present with the positive ions biased toward the more highly functionalized fullerenes and the negative ions biased toward the less highly functionalized fullerenes. Clearly, either the positive ion or the negative ion (or both) mass spectra are in error. Is there a way to identify which, if either, can be trusted?

The second column of Table 1 shows the calculated values of $\theta$ (using eq 11) for each dataset. For the positive mode $\theta$ is $\sim 2$, while for the negative mode $\theta$ is near 0.1 . Recall that a value of $\theta=1$ would demonstrate no bias in the data. For comparison, take the base- 10 $\operatorname{logarithm}$ of $\theta$. For the positive ion spectra $\log (\theta)$ equals 0.30 , and for the negative ion spectra $\log (\theta)$ equals -1 . Values of $\log (\theta)$ between 0.5 and -0.5 have, in our experience so far with polystyrene [3] and with resorcinarenes [12], indicated molecular mass distributions that can be corrected for bias.

Table 1 also shows the calculated values for $Q / k_{0}$ for each dataset. Applying these values via eq 17 to the positive and the negative data of the functionalized fullerenes shows very different results as seen in Figure 4. For the positive ion mode the data points lie on the $\mathrm{x}=\mathrm{y}$ line and the masses are evenly spaced on the axis as expected from the evenly spaced gravimetric mixtures prepared. In contrast, for the negative ion mode the data points do not lie on the line $\mathrm{x}=\mathrm{y}$ and there is still a clear bias in the spacing of the data points toward low mass. Thus, the Taylor's expansion of $k_{i}$ works well for the positively charged functionalized fullerenes where only a small correction is required but fails for the negatively charged fullerenes. As a final check of the results, estimates of the values for $\hat{k}_{1}$ and $\hat{k}_{2}$ are found from eq 12. Their ratio, $\theta$, is given in column 7 of Table 1 with the difference from the value estimated from eq 11 given in the last column. The small difference for the positive ion spectra indicates that the oligomer-byoligomer $k_{i}$ are consistent with the $Q / k_{o}$ found in the first part of the method. The large difference for the negative ion spectra indicates that the $k_{i}$ are inconsistent and the molecular mass distribution cannot be correct using only the first term in the Taylor's expansion.

Lastly, Figure 5 shows the raw and corrected molecular mass distributions for the high functionalized material in positive ion mode. The uncorrected values are simply the integrated peak intensities for the spectrum in the bottom panel of Figure 1. The corrected molecular mass distribution takes these values and multiplies them by the appropriate $k_{i}$. As expected, the higher mass ions are overcounted and their values are reduced accordingly while the lower mass ions have their relative intensities increased.

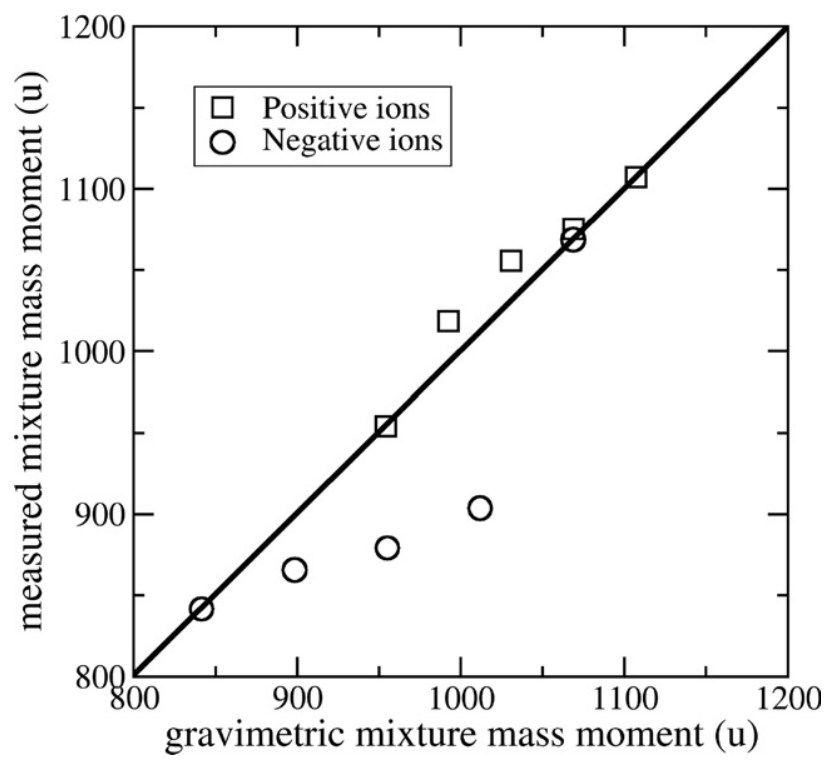

Figure 3. Mass-average molecular moments expected from the mixtures of the pure components (gravimetric) versus the actual values measured by mass spectrometry. Positive ion data from Figure 1 and negative ion data from Figure 2. The moments from the pure components by definition lie on the diagonal line $\mathrm{x}=\mathrm{y}$. The observation that all points do not lie on the diagonal line indicates that there is a mass bias in both ion modes. 
Table 1. Calculated values as described in the text

\begin{tabular}{|c|c|c|c|c|c|c|c|}
\hline Ion charge & $\theta$ & $Q / k_{o}$ & $M_{w 1}^{0}(\mathrm{u})$ & $M_{w_{2}}^{0}(\mathrm{u})$ & $M_{o}(\mathrm{u})$ & $\begin{array}{l}\theta \text { estimated from } \\
\mathrm{Q} / \mathrm{k}_{\mathrm{o}} \text { using eq } 12\end{array}$ & $\begin{array}{c}\% \text { Difference } \\
\text { in } \theta \\
\end{array}$ \\
\hline Positive & 1.92 & 0.00398 & 936 & 1101 & 1025 & 2.06 & $7 \%$ \\
\hline Negative & 0.15 & -0.00372 & 856 & 1095 & 950 & 0.34 & $120 \%$ \\
\hline
\end{tabular}

\section{Discussion}

With regard to the fulleropyrrolidines studied here, it may be inferred from these results that the error in molecular mass distribution measurement by mass spectrometry comes from differences in the ionization probability as a degree of functionalization. This is in contrast to errors that may arise from functional group loss, which has been observed previously for functionalized amphiphilic fullerenes [13]. In this work, differences in laser power did not show significant differences in molecular mass distribution as would be expected if functional groups were being removed by the ablation process. Likely, the errors are due to differences in analyte charging arising from differences in the number of functional groups. In positive ion mode, the proton forming the $\left[\mathrm{M}+\mathrm{H}^{+}\right.$ions are charged most likely on the ethylpyrrolidine functional group due to the well known proton affinity of pyrrolidines [14]. In the negative ion mode, the fullerene core is most likely to accept the negative charge to form the $\left[\mathrm{M}^{*}\right]^{-}$ion, which can be deduced from the large electron affinity of the fullerene $[15,16]$ as well as the electron donor-acceptor relationship of pyrrolidine-functionalized fullerenes [17]. including changes in this relationship due to the presence of multiple pyrrolidine functional groups. On this last point, Brustolon et al. [18] have shown by electron paramagnetic resonance that for

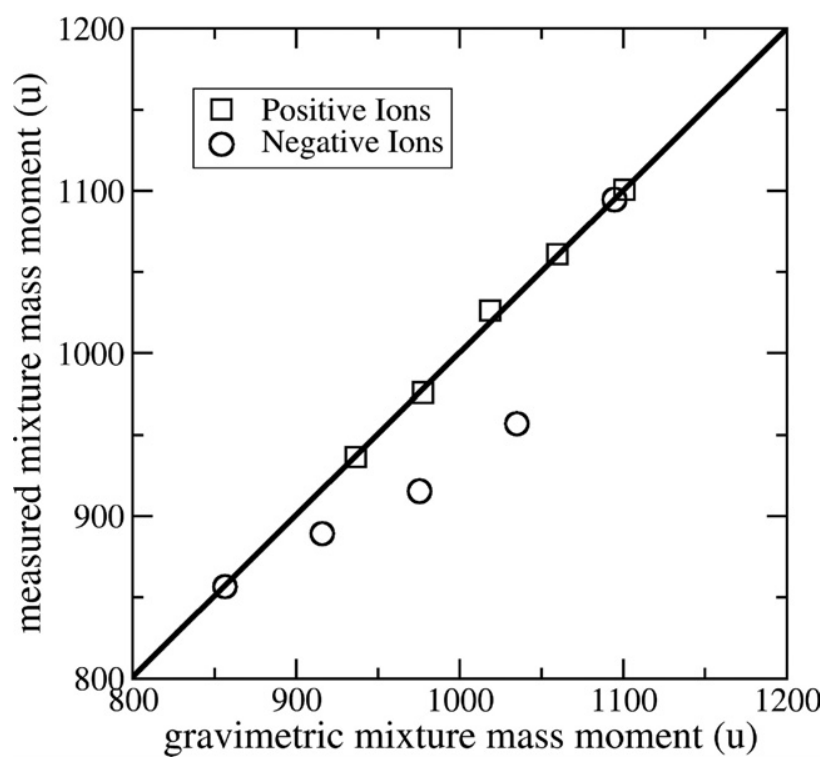

Figure 4. The gravimetric versus measured mass-average molecular moments from Figure 3 corrected using the optimal values of $Q / k_{0}$ calculated from eq 16 . mono- versus bis-substituted methylpyrrolidine fullerenes, the addition of more than one functional group breaks the electron symmetry of the fullerene anion. Breaking of the symmetry of the electron orbitals of the fullerene anion is likely to affect it electron affinity as well. In sum, assuming no cooperativity between the functional groups, the greater the multisubstitution the more opportunities to be charged positively, and the fewer opportunities to be charged negatively, leading to distortions in the measured molecular mass distribution. This is a result of the observation that the rate of in-plume reaction correlates with the exothermicity of the reaction [19]. Recent work on fluorofullerene mixtures [20] has shown reaction exothermicity to be a good predictor of MALDI-TOF mass spectral intensity.

With regard to the method presented, a truly comprehensive model of the $k_{i}$ would include the effects of the instrument settings, sample morphology, and the desorption/ionization process. However, such a physical model is beyond the current state of the art for competitive ion-molecule ionization reactions. Without such a comprehensive model, a simple approximation is used, specifically a Taylor's expansion model. A Taylor's expansion that is linear in mass creates a useful quantitative computational bridge from mass spectrum to molecular mass distribution. The mathematical model presented is far from exact, but appears to be robust provided a few conditions are met. Most importantly, for a given set of instrument parameters, and for a sample prepa-

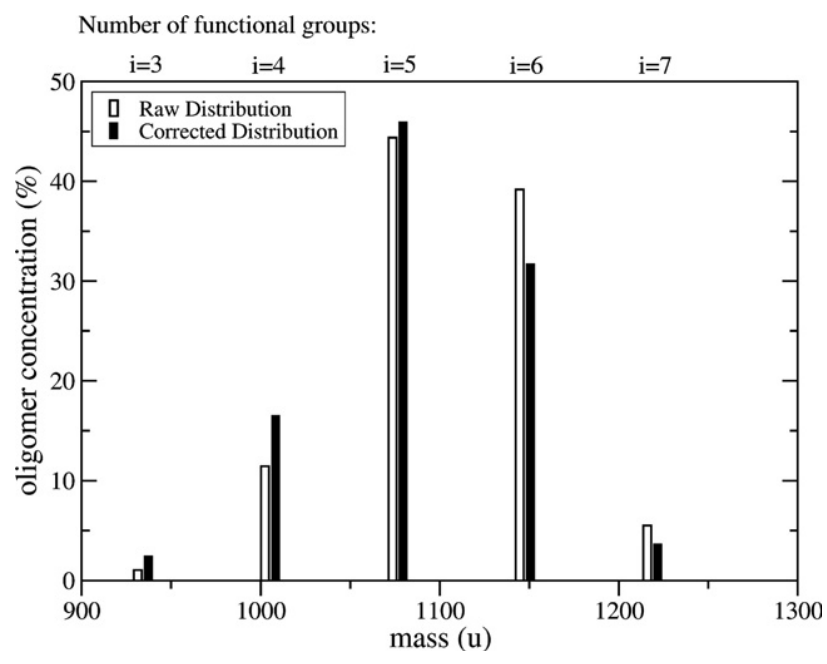

Figure 5. Uncorrected versus corrected MMD for the high functionalized positive ion data in Figure 1 using eq 17 and $Q / k_{0}$ from Table 1. 
ration with good homogeneity and repeatability, a fairly wide region of measurement parameter space, where the analyte-to-matrix mass ratio results in a proportional number of ions arriving at the detector must exist. This proportionality constant must be a slowing varying function of mass and oligomer concentration so that it can be linearly approximated. Additionally, this region of concentration space must have a high enough polymer concentration for us to obtain a good signal-to-noise ratio for statistically meaningful data analysis. Work in our laboratory continues on extending the mathematical model to expansion coefficients beyond the linear term, and on applying the Taylor's expansion method to different analytes and mass spectrometric methods.

\section{Conclusions}

A method to insure quantitative conversion of the MALDI-TOF mass spectra of polydisperse analytes to molecular mass distributions was presented. Mixtures of ethylpyrrolidine-derivatized fullerenes with different average molecular masses, coupled with a Taylor's expansion mathematical formalism, were used to ascertain the reliability of molecular mass distributions derived from mass spectra. Mass spectra of the derivatized fullerenes taken in positive ion mode were shown to give an accurate measured of the MMD which required only a small correction. Mass spectra of the derivatized fullerenes taken at the same time in negative ion mode did not provide accurate molecular mass distributions and could not be corrected. Differences in the mechanisms for ion formation are used to explain the discrepancy.

\section{Acknowledgment}

Certain equipment, instruments, or materials are identified in this article to adequately specify the experimental details. Such identification does not imply recommendation by the National Institute of Standards and Technology nor does it imply the materials are necessarily the best available for the purpose.

\section{References}

1. McLafferty, F. W.; Tureček, F. Interpretation of Mass Spectra, 4th ed.; University Science Books: Sausalito, CA, 1993, p 12.
2. Guttman, C. M.; Flynn, K. M.; Wallace, W. E.; Kearsley, A. J. A Report on the Certification of an Absolute Molecular Mass Distribution Polymer Standard: Standard Reference Material 2881; NIST Internal Report 7512, National Institute of Standards and Technology: Gaithersburg, MD, 2008. To download a copy, go to http://nvl.nist.gov/pub/nistpubs/ ir/2008/ir7512.pdf.

3. Guttman, C. M.; Flynn, K. M.; Wallace, W. E.; Kearsley, A. J. Quantitative Mass Spectrometry and Polydisperse Materials: Creation of an Absolute Molecular Mass Distribution Polymer Standard. Macromolecules 2009, 42, 1695-1702.

4. Hirsch, A.; Brettreich, M. Fullerenes-Chemistry and Reactions; WileyVCH: Weinheim, 2004.

5. Martín, N.; Altable, M.; Filippone, S.; Martín-Domenech, A. New Reactions in Fullerene Chemistry. SYNLETT 2007, 20, 3077-3095.

6. Bausch, J. W.; Surya Prakash, G. K.; Olah, G. A.; Tse, D. S.; Lorents, D. C.; Bae, Y. K.; Malhotra, R. Considered Novel Aromatic Systems. 11 Diamagnetic Polyanions of the C60 and C70 Fullerenes: Preparation, 13C, and 7Li NMR Spectroscopic Observation, and Alkylation with Methyl Iodide to Polymethylated Fullerenes. J. Am. Chem. Soc. 1991, 113, 3205-3206.

7. Prato, M.; Maggini, M. Fulleropyrrolidines: A Family of Full-Fledged Fullerene Derivatives. Acc. Chem. Res. 1998, 31, 519-526.

8. Lu, Q.; Schuster, D. I.; Wilson, S. R. Preparation and Characterization of Six bis(N-methylpyrrolidine)-C-60 Isomers: Magnetic Deshielding in Isomeric Bis adducts of C-60. J. Org. Chem. 1996, 61, 4764-4768.

9. Kordatos, K.; Da Ros, T.; Bosi, S.; Vazquez, E.; Bergamin, M.; Cusan, C.; Pellarini, F.; Tomberli, V.; Baiti, B.; Pantarotto, D.; Georgakilas, V.; Spalluto, G.; Prato, M. Novel Versatile Fullerene Synthons. J. Org. Chem. 2001, 66, 4915-4920.

10. Wallace, W. E.; Guttman, C. M.; Antonucci, J. M. Molecular Structure of Silsesquioxanes Determined by Matrix-Assisted Laser Desorption/ Ionization Time-of-Flight Mass Spectrometry. J. Am. Soc. Mass Spectrom. $1999,10,224-230$.

11. Hanton, S. D.; Hyder, I. Z.; Stets, J. R.; Owens, K. G.; Blair, W. R. Guttman, C. M.; Giuseppetti, A. A. Investigations of Electrospray Sample Deposition for Polymer MALDI Mass Spectrometry. J. Am. Soc. Mass Spectrom. 2004, 15, 168-179.

12. Wallace, W. E.; Flynn, K. M.; Guttman, C. M.; VanderHart, D. L Prabhu, V. M.; De Silva, A.; Felix, N. M.; Ober, C. K. Quantitative Measurement of the Polydispersity in the Extent of Functionalization of Glass Forming Calix[4]Resorcinarenes. Rapid Commun. Mass Spectrom. 2009, DOI: $10.1002 /$ rem.4099.

13. Brown, T.; Clipston, N. L.; Simjee, N.; Luftmann, H.; Hungerbühler, H. Drewello, T. Matrix-Assisted Laser Desorption/Ionization of Amphiphilic Fullerene Derivatives. Int. J. Mass Spectrom. 2001, 210/211, 249-263.

14. Hunter, E. P. L.; Lias, S. G. Evaluated Gas Phase Basicities and Proton Affinities of Molecules: An Update. J. Phys. Chem. Ref. Data 1998, 27, 413-656.

15. Brink, C.; Anderson, L. H.; Hvelplund, P.; Mathur, D.; Voldstad, J. D. Laser Photodetachment of C-60(-) and C-70(-) Ions Colled in a Storage Ring. Chem. Phys. Lett. 1995, 233, 52-56.

16. Guldi, D. M.; Prato, M. Excited-State Properties of C60 Fullerene Derivatives. Acc. Chem. Res. 2000, 33, 695-703.

17. Sun, Y. P.; Drovetskaya, T.; Bolskar, R. D.; Bau, R.; Boyd, P. D. W.; Reed C. A. Fullerides of Pyrrolidine-functionalized C-60. J. Org. Chem. 1997, $62,3642-3649$.

18. Brustolon, M.; Zoleo, A.; Agostini, G.; Maggini, M. Radical Anions of Mono- and Bis-Fulleropyrrolidines: An EPR Study. J. Phys. Chem. A 1998, 102, 6331-6339.

19. Knochenmuss, R. A Quantitative Model of Ultraviolet Matrix-assisted Laser Desorption/Ionization Including Analyte Ion Generation. Anal. Chem. 2003, 75, 2199-2207.

20. Streletskii, A. V.; Ioffe, I. N.; Kotsiris, S. G.; Barrow, M. P.; Drewello, T.; Strauss, S. H.; Boltalina, O. V. In Plume Thermodynamics of MALDI Generation of Fluorofullerene Anions. J. Phys. Chem. A 2005, 109, 714-719. 